\title{
MEDIDAS DE CONFIANZA MUTUA Y REACERCAMIENTO ENTRE CUBA Y LOS ESTADOS UNIDOS
}

\author{
Hal P. Klepak
}

Debido a los altibajos que experimentaron en los años setenta y ochenta la détente y las conversaciones sobre la misma, así como el control de armas y las conversaciones respectivas, volvió a cobrar vigencia una idea, seguramente muy antigua, relativa a la necesidad de que los países elaboraran medidas de confianza mutua. A partir de ese momento, y con ese nombre, esas iniciativas pasaron a ser objeto de numerosos estudios en los círculos diplomáticos y académicos. Denominadas confidence and security building measures (medidas de confianza mutua y seguridad) y conocidas por las siglas inglesas CBM o CSBM, estas iniciativas, que concitaron primeramente gran interés en las negociaciones que precedieron a los acuerdos relativos al c sntrol de armas y desarme en Europa, y más en general a los acuerdos sobre armas atómicas, fueron una de las piedras angulares del mejoramiento de las relaciones que, según se esperaba, habría de crear el marco estratégico para que la OTAN y el Pacto de Varsovia pudieran avanzar en una serie de aspectos relativos a la seguridad internacional.

Incluso el concepto se extendió durante la Conferencia sobre Seguridad y Cooperación Europeas, de manera de que pudiesen ser incorporados a esos convenios aquellos estados neutrales que deseaban participar en la construcción de un nuevo y más amplio sistema de seguridad europea. De ese modo, las CBM pasaron a ser concebidas en general como un conjunto de iniciativas explícitamente encaminadas a: contribuir a clarificar las intenciones militares de los países signatarios de esos convenios, y/o disminuir la incertidumbre derivada de las actividades militares, y/o reducir las posibilidades de hacer uso de la fuerza militar. ${ }^{1}$ Los procedimientos incorporados a las CBM tienen como propósito aliviar las tensiones y aminorar el peligro de que se desate un conflicto armado como consecuencia de una mala

\footnotetext{
${ }^{1}$ Las definiciones que se emplean en este artículo coinciden en general con las que utiliza el
} Canadian Department of External Affairs en los asuntos relativos a la seguridad. 
interpretación o un error de cálculo acerca de las maniobras de un estado rival.

Tan provechosa ha sido la aplicación de las CBM en Europa durante los últimos años, que ha comenzado a estudiarse cada vez más a fondo la posibilidad de trasladar el concepto mismo y las actividades conexas a otras regiones del mundo. Afortunadamente no se ha pasado por alto el carácter específico de la situación europea y, por consiguiente, la imposibilidad de tomarla sin más como "modelo" para las restantes regiones del globo. No obstante, numerosos autores de América Latina, Asia y Africa han tratado de sacar de esa situación particular conclusiones que podrían servir en sus respectivos continentes. ${ }^{2}$ Esta corriente cobró especial vigor desde que el Secretario General de las Naciones Unidas, Boutros Boutros-Ghali, en el documento titulado Agenda para la Paz, hizo un llamado a las organizaciones regionales para que pusieran más empeño en la preservación de la paz, la pacificación y en general el arreglo pacífico de las controversias.

Tanto se ha extendido, en efecto, el empleo de estas medidas dentro y fuera de Europa, que un autor se refirió a su aplicación como "el mecanismo fundamental para evitar el estallido accidental de una guerra o el agravamiento involuntario de las tensiones en las zonas sometidas a conflicto" durante el período de la post Guerra Fría. ${ }^{3}$ Es más, aun cuando las medidas puedan ser formales o informales, tácitas o ampliamente publicitadas, siempre nacen en último término de la voluntad política de las partes para aliviar las tensiones y crear un ambiente apropiado para al menos aumentar el grado de confianza mutua y cooperación prevaleciente hasta ese instante, cosa que es posible lograr con la aplicación de las CBM sin que los estados descuiden su propia seguridad.

Estas iniciativas deben considerarse, además, dentro de un contexto mucho más vasto y como un elemento clave, aunque sólo uno, de entre una amplísima gama de instrumentos encaminados a intensificar la seguridad, entre los cuales figuran la diplomacia preventiva, la pacificación, la observación de la paz, la creación de zonas

\footnotetext{
${ }^{2}$ Comisión Sudamericana de Paz, El sentido de una tarea, (Santiago: Andros, 1990), p. 52; Hernán Patiño Mayer, "Aportes a un nuevo concepto de seguridad hemisférica-seguriđad cooperativa", Seguridad estratégica regional, N24, septiembre de 1993, pp. 84-89; y Hugo Palma, América Latina: limitación de amamentosy desarme en la región, (Lima: CEPEI, 1986), pp. 58-62.

${ }^{3}$ Michael Krepon, "The Decade for Confidence-Building Measures", en: Michael Krepon et a1. (eds.), A Handbook for Confidence-Building Measures for Regional Security, (Washington, D. C.: Henry L. Stimson Center), Handbook Ne1, p. 1.
} 
de paz, la conservación de la paz, la puesta en vigor de la paz y las así denominadas medidas para evitar conflictos, que suelen constituir el paso inmediatamente precedente a la aplicación plena de las CBM, cuyo único e importante propósito consiste en alejarse de una situación conflictiva e iniciar, a partir de ahí, el largo proceso que supone llegar a un nivel superior de confianza mutua.

Aunque por cierto no es imprescindible que las CBM tengan carácter militar, el papel central que han ocupado las cuestiones militares en los asuntos relativos a la seguridad ha hecho prevalecer en general ese aspecto en la mayoría de las regiones en conflicto. No obstante, dos países potencialmente adversarios pueden recurrir a otras vías para reducir la desconfianza, sobre la base de adoptar una posición común respecto a un número considerable de problemas y cooperar por ejemplo en temas relativos al comercio, la salud, el medio ambiente, la política exterior, la investigación científica y un sinfín de otros campos. Con todo, pese a lo variadas y múltiples que puedan ser esas iniciativas, no hay que perder de vista que su objetivo fundamental es de carácter político, esto es, consiste en crear un clima esencialmente político de mayor confianza entre los líderes de uno y otro país. ${ }^{4}$ Las relaciones políticas entre las partes que suscriben un acuerdo de CBM deben mejorar día a día, y eso exige que el modo en que los líderes perciben a sus interlocutores experimente un vuelco, de manera que éstos, antes negativos y hostiles, comiencen a aparecer como elementos positivos y menos escépticos.

Es preciso tener en cuenta, no obstante, que en general las CBM han conseguido sus mejores resultados cuando han llegado a formar parte de un proceso político ya en marcha, de modo que, aunque han demostrado ser un excelente medio para acelerar y profundizar el mejoramiento de las relaciones, es muy poco probable que puedan operar como elemento catalizador de todo el proceso desde su inicio.

El Departamento Canadiense de Relaciones Exteriores ha clasificado las CBM en dos categorías: las CBM de información y comunicaciones y las CBM restrictivas. Las primeras comprenden la disponibilidad de:
- información acerca de las fuerzas, instalaciones, estructuras y actividades militares;
- sistemas de comunicación;

\footnotetext{
${ }^{4}$ Edgardo Mercado Jarrín, "Perspectivas de los acuerdos de limitación y desarme en América Latina y el Caribe", en: Augusto Varas (ed.), Paz, desarme y desarrollo en América Latina, (Buenos Aires: GEL, 1987), pp. 309-311.
} 
- notificación anticipada de determinadas actividades militares; y

- oportunidades para observar determinadas actividades militares.

Las CBM restrictivas incluyen la disponibilidad de:

- oportunidades para "inspeccionar" y/o monitorear fuerzas militares limitadas o restringidas, instalaciones, estructuras y actividades;

- no interferencia con las medidas de verificación;

- seguridad de evitar o limitar actividades militares provocativas;

- seguridad de evitar o limitar el "estacionamiento" o "emplazamiento" de fuerzas militares provocativas; $y$

- seguridad de evitar o limitar el desarrollo o despliegue de determinadas "tecnologías" militares, incluyendo los sistemas o subsistemas que los Estados partes estimen de carácter o impacto desestabilizador.

Una vez definidas las CBM en sus aspectos generales, es posible comenzar a situarlas en el contexto de las actuales relaciones entre Cuba y los Estados Unidos, a fin de considerar si tiene sentido buscar unas $\mathrm{CBM}$ que pudieran reducir la tensión y crear un ambiente político más propicio para mejorar las relaciones entre La Habana y Washington. También será necesario analizar si no existen otros caminos, distintos del que definen estas iniciativas, para iniciar un proceso de reacercamiento que las CBM futuras podrían posteriormente acelerar o consolidar.

\section{Las relaciones actuales entre Cuba y Estados Unidos.}

No es aventurado decir que las relaciones entre ambos países están tan deterioradas como siempre o incluso en el peor momento de su casi permanente historia turbulenta. ${ }^{5} \mathrm{~A}$ pesar de las ilusiones que se habían hecho algunos sectores en el sentido de que el gobierno de Clinton daría un nuevo enfoque al tema, lo cierto es que las relaciones han empeorado en el curso de los primeros meses de gobierno. La Cuban Democracy Act asestó un durísimo golpe a quienes deseaban reabrir el diálogo entre los dos países, al tiempo que el embargo y las medidas militares que Estados Unidos despliega contra Cuba, lejos de atenuarse, han recrudecido durante el actual gobierno.

\footnotetext{
${ }^{5} \mathrm{La}$ evolución de estas relaciones durante los primeros 25 años del gobierno de Castro está descrita en: Wayne S. Smith, The Closest of Enemies, (Nueva York: Norton, 1987).
} 
El único cambio verdadero consiste en que, a causa de la desesperada situación en que la ha dejado el fin de la Unión Soviética y del Pacto de Varsovia, Cuba se ha visto obligada a revisar muchos aspectos de su política exterior, y a adoptar una actitud de absoluta no provocación con respecto a Washington en todos los temas importantes que existen entre ambos países. No es difícil imaginar la afrenta que debe significar para los sectores más radicalizados del gobierno y del partido una actitud tan humillante, pero la realpolitik oblige.

La Habana percibe que Estados Unidos no se ha desviado un ápice del propósito de aislar, debilitar y, de ser posible, derrocar al gobierno de Castro, pese a que Cuba no representa ya ni la más mínima amenaza para ese país o sus intereses en general. Según las palabras del propio Castro, Estados Unidos, dejándose llevar por un sentimiento "triunfalista", no está dispuesto a ceder en ningún aspecto importante mientras siga gozando de su posición privilegiada en el nuevo mundo unipolar. La consigna es, pues, defenderse contra lo que se anuncia como la amenaza cada vez mayor de invasión, subversión o una combinación de ambas proveniente del Norte.

Por su lado, Estados Unidos ve al gobierno de Castro como un anacronismo que trata desesperadamente de subsistir después del colapso del movimiento comunista internacional; como una fruta podrida a punto de caer del árbol. Según esta percepción, el régimen de Castro va sencillamente a recibir su merecido por las largas décađas de opresión, explotación y exportación de la revolución, y Estados Unidos debe contribuir a su manera a que ello ocurra, aunque no en forma demasiado directa, porque, al menos esta vez, no hay necesidad alguna de precipitar el curso natural de los acontecimientos.

Dentro de este panorama, reviste particular importancia someter a un nuevo análisis los aspectos de seguridad implícitos en las relaciones cubano-estadounidenses. Durante mucho tiempo, la amenaza norteamericana fue la justificación fundamental de los enormes esfuerzos que desplegó Cuba para hacerse de un formidable aparato militar. Antes de las últimas reducciones de personal, las Fuerzas Armadas Revolucionarias tenían 175.000 hombres en servicio regular, a lo que es preciso agregar el muy considerable contingente de la reserva y de las organizaciones paramilitares: 100.000 hombres en el Ejército Juvenil del Trabajo; 50.000 en la Fuerza de Defensa Civil; 15.000 en la Seguridad del Estado; 4.000 en el Servicio de Fronteras, 
más una gigantesca Milicia Territorial integrada por 1.300 .000 hombres. ${ }^{6}$ La subversión fomentada por los Estados Unidos dio pie asimismo para crear otro enorme servicio de seguridad interna, dentro del cual se cuentan los ahora muy despretigiados Comités de Defensa de la Revolución. ${ }^{7}$

De manera semejante, aunque menos aparatosa, Estados Unidos se valió de la "amenaza" cubana, en conjunto con la de la Unión Soviética, para justificar no sólo la creación de un sistema de seguridad hemisférico de vastas proporciones, sino más específicamente, el despliegue en gran escala de fuerzas militares (y de iniciativas diplomáticas de la más variada índole) en la zona del Caribe. ${ }^{8}$ Con la excusa de que Cuba constituía una cabeza de playa para la subversión comunista en América Latina, Estados Unidos reforzó todo el dispositivo de seguridad interamericano y lo transformó en un aparato de seguridad interna en cada uno de los respectivos países. ${ }^{9}$ Según la lógica consistente en ponerse en el peor de los casos, se llegó incluso a sostener que en tiempo de guerra, Cuba podía servir a la Unión Soviética como base de operaciones decisivas en contra de los buques norteamericanos y aliados que surcaran el Golfo de México y el Caribe, y que las propias fuerzas armadas cubanas podían ser de gran ayuda para atacar los blancos occidentales situados en esas zonas. ${ }^{10}$ Más aún, aunque muchos análisis han ridiculizado esas afirmaciones, los sectores interesados en mantener la tensión entre ambos países siguen recurriendo una y otra vez a ellas. ${ }^{11}$

\footnotetext{
${ }^{6}$ International Institute of Strategic Studies, The Military Balance 1993-1994, (Londres: Brassey's, 1993), pp. 182-183; y Adrian English, The Armed Forces of Latin America, (Londres: Jane's, 1984), pp. 199-218.

${ }^{7}$ Andrés Sorel, Cuba: la revolución crucificada, (Madrid:Libertarias/Prodhufi), pp. 117-122.

${ }^{8}$ Véase el excelente trabajo de Lars Schoultz, National Security and United States Policy toward Latin America, (Princeton, Nueva Jersey: Princeton University Press, 1987); y Pierre Queuille, L'Amérique-latine, la doctrine Monroe et le panaméricanism, (París: Payot, 1969), pp. 220-236.

${ }^{9}$ Alain Rouquié, L'état militaire en Amérique-latine, (París: Seuil, 1982), pp. 148-189.

${ }^{10}$ Jaime Suchlicki (ed.), The Cuban Military under Castro, (Miami: University of Miami Press, 1989), pp. 168-171; y Michael C. Desch, When the Third World Matters: Latin America and United States Grand Strategy, (Baltimore: Johns Hopkins University Press, 1993), pp. 120-122.

${ }^{11}$ Schoultz, op. cit.,pp. 260-265; y Abraham Lowenthal, Partners in Conflict: The United States and Latin America in the 1990's, (Baltimore: Johns Hopkins University Press, 1990), pp. 164-167. Véase también Ivelau L. Griffith, The Quest for Security in the Caribbean, (Armonk, Nueva York: M. E. Sharpe Publishers, 1993), pp. 29, 190 y 192.
} 


\section{El cambio.}

Parece evidente que en la actualidad carece de toda validez, si es que alguna vez la tuvo, el alcance estratégico que Cuba y los Estados Unidos, cada cual por su lado, atribuyeron a las amenazas. Como se acaba de indicar, existe la impresión generalizada de que el gobierno de Castro está a punto de desplomarse a causa de sus propias contradicciones, de modo que muy pocas personas en los Estados Unidos, aparte de la muy ruidosa pero reducida comunidad cubana en el exilio, creen que sea preciso acelerar la caída mediante una intervención militar directa, con todo el peligro para los soldados y los intereses estadounidenses que ello necesariamente llevaría aparejado. Así piensa en especial el Pentágono, al parecer firmemente convencido de que un ataque contra Cuba sería no sólo imprudente sino prácticamente ilógico. Aunque ciertamente sería insensato que los encargados de la seguridad cubana no tomaran en serio la posibilidad de una invasión o de un movimiento subversivo, sobre todo teniendo en consideración el aumento del poderío bélico norteamericano en el último tiempo, no hay que olvidar que una amenaza real está constituida por la capacidad de atacar y por la intención de hacerlo, elemento este último que parece claramente ausente de la política estadounidense actual.

Es sencillamente imposible que Estados Unidos siga tomando en serio la amenaza cubana. Las Fuerzas Armadas de este país se han reducido en forma muy considerable y carecen en general de combustible, repuestos, municiones para el adiestramiento o la defensa, e incluso de perspectivas de futuro. Sin el apoyo del Pacto de Varsovia, su poderio bélico ha menguado en forma notoria y seguirá declinando, en especial en lo referente a la capacidad ofensiva. Por su parte, las fuerzas norteamericanas, aunque también han sido reducidas, se han desplegado ahora en la zona del Caribe, incluso en mayor número que durante la Guerra Fría, y las Fuerzas Armadas Revolucionarias se ven en este momento aventajadas en todos los aspectos imaginables en cuanto a poderío militar, aun dentro de la zona del Caribe; ello sin siquiera tener en cuenta los vastos recursos que Washington podría poner en juego si decidiera hacer algo más concreto acerca de la situación de la Isla.

La situación de seguridad de Cuba y los Estados Unidos ha experimentado, sin embargo, un cambio profundo, y es posible, e incluso probable, que más de un problema importante derivado de 
las nuevas circunstancias llegue a afectár a ambos países. En efecto, la principal "amenaza" no está representada ahora por el ataque directo de uno u otro, ni por las complicaciones externas, sino más bien por la posibilidad de que los cambios que tengan lugar en Cuba escapen de todo control y desemboquen en un estallido de violencia que envuelva toda la Isla. En otras palabras, existe la clara posibilidad de que los exiliados o incluso los propios habitantes de la Isla traten de derrocar el régimen por la fuerza. También es posible que éste se vea obligado a recurrir a la fuerza para controlar el descontento de la población, lo cual no haría otra cosa que radicalizar a la oposición y contribuir al aumento de la violencia.

No es inverosímil que una situación de esta naturaleza pueda tener consecuencias sumamente graves para la seguridad de uno y otro país. Es más que improbable que los exiliados vayan a permanecer indiferentes si los acontecimientos toman este curso; sin embargo, lo más importante es que existe el serio peligro de que el desorden generalizado, la violencia y el derramamiento de sangre se conviertan en una guerra civil declarada, y entonces sería inevitable que Estados Unidos se sintiera llamado a intervenir para poner fin a la lucha y restablecer la paz, y ciertamente también para instalar un nuevo gobierno. En caso de que la violencia se prolongara, la emigración crecería a pasos agigantados, lo que no dejaría de tener graves consecuencias para el país del Norte, sin duda el principal destino de la emigración, que en el último tiempo ha tenido experiencias muy gráficas al respecto.

Así, pues, es de interés para ambas naciones cuidar que los cambios que tengan lugar en la Isla ocurran en forma gradual, controlada y sin derramamiento de sangre. Esto no quiere decir que los dos gobiernos consideren la situación actual en esos términos; sólo se pretende señalar que, en beneficio de todas las partes, hay razones más que suficientes para considerar las cosas de esta manera.

\section{Situación actual de las CBM.}

Antes de analizar el aporte que podrían hacer las CBM al mejoramiento de las relaciones entre ambas naciones, es importante referirse a los problemas de asimetría: Cuba es un país pequeño y actualmente más débil que en muchas décadas con respecto a los Estados Unidos. Este, en cambio, es la potencia dominante (y quizá la única potencia) 
del mundo absolutamente sin rivales en cuanto a hegemonía política y militar, no sólo en Occidente sino en la comunidad internacional, ni más ni menos.

Ello significa que Estados Unidos está muchísimo más capacitado que ningún otro para mostrarse dispuesto a iniciar un proceso de acercamiento; sin embargo, dada su actitud de extraordinario y efectivo triunfalismo, es, con mucho, el país menos motivado para hacerlo. Cuba, pequeño y débil, es, con creces, el menos capacitado para hacer gestos de ese tipo con respecto a los Estados Unidos, pero está obligado a hacerlo si ha de lograrse algún avance en la materia. Se trata ciertamente de una situación injusta, pero como ya lo hizo ver Tucídides, las relaciones internacionales, especialmente las que median entre los países poderosos y los débiles son esencialmente injustas.

Lo que se precisa fundamentalmente es que Estados Unidos preste atención a Cuba, cosa relativamente difícil en este momento, dado que el programa de relaciones exteriores del gobierno de Clinton está, por decir lo menos, completamente copado. La Habana debe esforzarse por hacer comprender a Washington que no hay nada que perder, y sí mucho por ganar, si ambos países abordan derechamente la nueva situación de seguridad y tratan en conjunto de evitar los conflictos o entrar en una situación que pueda degenerar en violencia y, por ende, en movimientos populares e intervención norteamericana. El gobierno de Castro debe demostrar al gobierno estadounidense que de verdad está cambiando en aspectos importantes, y que es de interés de ambos gobiernos que ese cambio transcurra sin demasiados altibajos. Al hacer eso, el gobierno cubano debe esforzarse en demostrar al mismo tiempo que es un interlocutor válido y digno de confianza. Estas iniciativas podrían preceder a la aplicación más amplia de las CBM, pero pueden incluso considerarse como la base sobre la cual han de cimentarse esas medidas.

Cuba debe ser capaz de emplear el arma más eficaz con que puede contar un país que trata con los Estados Unidos: la opinión pública. Las encuestas ya están mostrando que una gran mayoría de los ciudadanos de este país no creen que deba recurrirse a la fuerza para desplazar al gobierno de La Habana. Es necesario capitalizar ese sentimiento, poniendo en práctica desde ya medidas de confianza mutua, de carácter inicial y unilateral, antes de pasar al despliegue de otras de índole propiamente bilateral. 
Es importante tener en cuenta que, gracias a un trabajo de muchos años, ambos países ya han aplicado algunas de esas medidas, las que, aunque no han logrado resultados decisivos, han funcionado hasta ahora razonablemente bien. Nos referimos, por ejemplo, a los acuerdos sobre piratería aérea y tráfico de drogas. Incluso se han suscrito algunas CBM de carácter militar, relativas esta vez a la navegación en alta mar y especialmente a la base estadounidense de Guantánamo. Dado el pequeño tamaño de la base y habida cuenta de las características de la bahía en que está situada, es imprescindible notificar por adelantado cualquier movimiento naval. Como se trata de un problema práctico, los militares norteamericanos se han mostrado más que dispuestos a tratar con las Fuerzas Armadas cubanas para procurar reducir toda posibilidad de conflicto que pudiera surgir de un malentendido o de un error de apreciación. Ninguno de los dos bandos ha querido dar pie a esos errores, y ambos se han preocupado de eliminar todos aquellos elementos que pudieran originarlos. Este es el modo en que operan normalmente las CBM en el resto del mundo, lo que muestra su grado de utilidad y flexibilidad. ${ }^{12}$

Cuba debería esforzarse al máximo por dar a conocer al pueblo estadounidense la existencia de esos acuerdos y de esa forma de cooperación. En materia de seguridad, debe además dar pruebas de su buena voluntad en lo que concierne a los recelos que todavía se anidan en Washington, por infundados que éstos sean, dando para ello pasos tendientes a reducir aquellos elementos bélicos que Estados Unidos consideraba antes como una amenaza; además, debe hacer todo esto con un gran despliegue simultáneo de relaciones públicas. Gran parte de esto puede llevarse a cabo sin que Cuba vea debilitada para nada su defensa.

Por ejemplo, el gobierno estadounidense ha mostrado una y otra vez su preocupación por la amenaza que representan las fuerzas anfibias cubanas para la zona del Caribe. La Marina de Cuba cuenta con dos Polnocny (buques anfibios de desembarco), cada uno de ellos capaz de transportar seis tanques y 180 hombres, y dos batallones de infantería de marina, cuya principal misión en tiempos de guerra tendría que ver con operaciones anfibias. ${ }^{13} \mathrm{Sin}$ embargo, la red

\footnotetext{
${ }^{12}$ Una descripción del modo en que han funcionado las CBM en América Central, y que han tenido que ver con los intereses que los Estados Unidos consideran sumamente importantes, aparece en la interesantísima contribución de Jack Child, The Central America Peace Process 1983-1991, (Boulder, Colorado: Lynne Rienner, 1992), pp. 151-157.

${ }^{13}$ International Institute of Strategic Studies, op. cit, p. 183; y Suchlicki, op. cit, p. 168.
} 
caminera cubana es mucho mejor que la de la mayoría de los países latinoamericanos, lo que asegura que sus Fuerzas Armadas puedan llegar a casi todos los puntos del territorio con razonable rapidez y eficiencia, de manera que resulta en verdad cuestionable la utilidad que pudieran tener esos buques para la defensa de la Isla. En segundo lugar, en caso de guerra, la superioridad aérea y naval que tendría Estados Unidos sería tan aplastante, que resultaría inconcebible que esos barcos pudieran hacerse a la mar y actuar con eficacia. Por consiguiente, Cuba perdería muy poco si eliminara esas naves o, mejor aún, si las devolviera a Rusia y lo hiciera además con el mayor despliegue publicitario.

Prácticamente lo mismo puede decirse de la flota de transporte aéreo de largo alcance de la Fuerza Aérea de Cuba, en particular del IL-76, transporte militar de gran fuselaje. Aunque puede argumentarse que el transporte aéreo de corto alcance es decisivo para defender el país de una invasión (pese a que en tal caso el poderío aéreo estadounidense podría perfectamente inutilizarlo), hay que convenir en que el de largo alcance es mucho menos vital. Numerosos observadores norteamericanos han denunciado que Cuba sigue abrigando oscuros designios acerca de sus vecinos o de países muy distantes de reciente interés ideológico. Cuba podría ganar muchísimo en el terreno de las relaciones públicas si devolviera esos aviones a Moscú, pues así estaría mostrando que ya ha dejado atrás todo aquello de exportar la revolución y de perturbar a otros países. Una. iniciativa de esa naturaleza prácticamente no debilitaría para nada la capacidad defensiva de la Isla.

Obligada por la desastrosa situación económica, Cuba ha debido reducir considerablemente sus Fuerzas Armadas en el último tiempo. Esas reducciones se llevaron a cabo calladamente, casi como un acto vergonzoso. Por el contrario, quizá lo más conveniente habría sido mostrarlas abiertamente, como una prueba más de que Cuba está cambiando, que se está desmilitarizando, que ha perdido interés en cualquier aventura extranjera y que está segura de no enfrentar ningún problema interno serio. Estados Unidos conoce bien la magnitud de esas reducciones y el efecto que han tenido en el tamaño de las Fuerzas Armadas. Por consiguiente, nada debería impedir que se aprovechara esa situación directamente desde el punto de vista de las relaciones públicas. Además, al hacerlo, se podría poner de relieve el carácter pacífico de las intenciones cubanas, en contraste con las connotaciones muy deshonrosas que tiene la situación para los nor- 
teamericanos: la lucha entre David y Goliat, en que éste último golpea al que ha caído al suelo.

Finalmente, pese a las repetidas acusaciones en sentido contrario, Cuba puede exhibir resultados muy buenos en materia de lucha contra el narcotráfico, al menos en comparación con los Estados Unidos y sus aliados. ${ }^{14}$ Podría, pues, hacer un mayor esfuerzo para comprometerse, y mostrar públicamente que desea unirse, a las operaciones que se llevan a cabo en el Caribe. Las Fuerzas Armadas y la policía de varios países aliados de los Estados Unidos (entre ellos Gran Bretaña, Francia, los Países Bajos y la mayoría de las naciones del Caribe) participan activamente en esas operaciones. En las aguas y cielos que circundan a Cuba es donde mayor cooperación necesitan los vecinos, especialmente los territorios vinculados a Gran Bretaña (las Islas Turcas y Caicos por el Norte, y las Islas Caimán por el Sur), Bahamas y Jamaica. ${ }^{15}$ Aunque a juzgar por algunos graves errores (como el hundimiento, en 1980, del patrullero Flamingo de la Real Fuerza de la Defensa de Bahamas por parte de un grupo de Mig cubanos), Cuba tendrá todavía que desplegar prolongados esfuerzos para ganarse la confianza de esos vecinos, el hecho de llegar a una buena relación con ellos constituiría una verdadera jugada maestra para alcanzar otro nivel de cooperación indirecta con los Estados Unidos en materia de seguridad, lo que a la larga podría dar muy buenos dividendos. ${ }^{16}$ De hecho, en septiembre de 1991, Cuba y Bahamas iniciaron conversaciones precisamente en torno a la posibilidad de cooperar en tal sentido. ${ }^{17}$

Las iniciativas militares señalađas, algunas de las cuales pueden considerarse una suerte de CBM, no son las únicas que pueden presentarse. En el terreno diplomático, La Habana ha admitido que debe adoptar una política no confrontacional con respecto a los Estados Uniclos. Sin embargo, seguramente es necesario dar mayor publicidad a esa decisión; bien podría utilizarse a los grupos partidarios de Cuba para difundir en los Estados Unidos y demás países occidentales las nuevas tendencias de la política exterior cubana.

En lo económico, la apertura debería ampliarse, y quizá más rápidamente que hasta ahora. El régimen de Castro también podría

\footnotetext{
${ }^{14}$ Peter D. Scott y Jonathan Marshall, Cocaine Politics: Drugs, Armies and the CIA in Central America, (Berkeley, California: University of California Press, 1991), pp. 102-103.

${ }^{15}$ Este tema se examina en Griffith, op. cit., pp. 243-271.

${ }^{16}$ Ibid., p. 200.

${ }^{17}$ Ibid, p. 270.
} 
mejorar su imagen en lo relativo al espinudo asunto de los derechos humanos, y mostrar progresos para no presentar un flanco al ataque de sus enemigos. También en este caso es necesario exhibir en detalle esos avances y darles amplia publicidad.

\section{Justicia y conclusiones.}

Ciertamente, sería fácil argüir que Cuba no está en absoluto obligada a dar ninguno de esos pasos: es un país soberano y como tal tiene pleno derecho a contar con las Fuerzas Armadas y a desplegar la política exterior que desee, y llevar a cabo los cambios internos conforme al modo en que lo decida su gobierno. Nada de eso es objetable.

No obstante, con el propósito de avanzar hacia una situación en que las CBM puedan llegar a ser efectivamente bilaterales, es difícil no llegar a la conclusión de que en el momento actual La Habana debe dar mayores muestras de flexibilidad que Washington. Estado Unidos no ha avizorado aún, al menos públicamente, los problemas que podría acarrearles el hecho de no llegar a un mayor grado de cooperación con Cuba. Como ya se señaló, las CBM sirven más para hacer avanzar un proceso de reacercamiento ya en marcha que para darle inicio. Sin embargo, en las actuales circunstancias, parece efectivamente que las decisiones unilaterales que eventualmente adopte La Habana (de poco costo, pero quizá fructíferas) podrían aplicarse después de las CBM que ya están en curso, y allanar el camino para aliviar la situación desesperada que afronta actualmente la Isla, la que si no es manejada en forma apropiada, podría tener a futuro los efectos más negativos que es dable imaginar. 